\title{
Short-term complications and long-term morbidities associated with repeated unplanned extubations
}

\author{
Leeann R. Pavlek $\mathbb{1}^{1,2} \cdot$ Julie Dillard ${ }^{3} \cdot$ Gregory Ryshen ${ }^{4} \cdot$ Emily Hone $^{5} \cdot$ Edward G. Shepherd $\mathbb{D}^{6} \cdot$ \\ Mohannad Moallem ${ }^{5}$
}

Received: 2 March 2020 / Revised: 6 November 2020 / Accepted: 15 January 2021 / Published online: 5 February 2021

(c) The Author(s), under exclusive licence to Springer Nature America, Inc. 2021

\begin{abstract}
Objective To describe characteristics associated with repeated unplanned extubations, short-term complications and outcomes, and longer-term morbidities including acquired subglottic stenosis.

Study design Cohort study including neonates admitted to a tertiary care neonatal intensive care unit who experienced an unplanned extubation in a 5-year period.

Results We reviewed 588 events involving 300 patients. Ten percent had airway trauma with reintubation, $42 \%$ required $\geq 2$ reintubation attempts, and 39\% led to increased baseline oxygen. Increased odds of repeated events were seen in patients with bronchopulmonary dysplasia and were associated with higher rates of tracheostomy and longer length of stay. The 9\% of patients diagnosed with acquired subglottic stenosis had more unplanned extubations, higher rates of airway trauma and tracheitis, and were an older gestational age at birth.

Conclusion Unplanned extubations lead to short- and long-term morbidities. Certain patient characteristics are associated with increased odds of repeated events and the development of acquired subglottic stenosis.
\end{abstract}

\section{Introduction}

An unplanned extubation (UE) is defined as the unintentional dislodgement of an endotracheal tube (ETT) from a patient receiving invasive mechanical ventilation [1]. In North American neonatal intensive care units (NICUs), UE requiring reintubation is the fourth most common adverse

Leeann R. Pavlek

Leeann.Pavlek@Nationwidechildrens.org

1 Division of Neonatology, Nationwide Children's Hospital, Columbus, $\mathrm{OH}$, USA

2 Center for Perinatal Research, Abigail Wexner Research Institute at Nationwide Children's Hospital, Columbus, OH, USA

3 Department of Pediatrics, Saint Louis University, St. Louis, MO, USA

4 Quality Improvement Services, Nationwide Children's Hospital, Columbus, $\mathrm{OH}$, USA

5 Biostatistics Resource at Nationwide Children's Hospital, Columbus, OH, USA

6 Neonatal Intensive Care Department, King Abdulaziz Medical City, Riyadh, Saudi Arabia safety event [2]. Reported rates of UE in the NICU vary between 0.56 and 5.3 UEs per 100 ETT days [3, 4]. In a review of the adverse events related to intubations in a tertiary care NICU, four out of ten endotracheal intubations were associated with adverse events. A greater than fourfold increased odds of an adverse event was seen if the intubation was performed emergently, with UE found to be the most common reason for an emergent intubation [5]. Other studies demonstrated a $20 \%$ rate of cardiovascular collapse following UE in NICU, pediatric ICU, and pediatric cardiac ICU patients [6].

The risk of UE is generally higher in neonates when compared to older patients, likely due to longer duration of intubation, shorter trachea length, low use of routine sedation, use of uncuffed ETTs, and encouragement of skin-toskin care [1]. Several studies have reported on the specific risk factors for UEs, such as agitation, poor fixation of the tube, and duration of mechanical ventilation. Reported rates of reintubation following UE range from 8.3 to $100 \%$, though there are little data regarding risk factors in neonates for requiring reintubation or the use of sedation and/or restraints [2, 3, 7-9]. A recent single-center cohort study reported that the use of a sedation infusion, high-frequency ventilation, higher mean airway pressure, and need for 
cardiopulmonary resuscitation were associated with immediate reintubation following UE without a trial of extubation [10]. Despite studies investigating UEs in general, there is little evidence in the literature about the risk factors for repeated UEs or the short- and long-term outcomes associated with these events in the NICU, except for one case-control study of severe acquired subglottic stenosis (SGS) in neonates that reported the incidence of repeated UEs in these patients [11].

In this study, we aim to describe a 5-year cohort of patients who experienced a UE, focusing on risk factors contributing to UE and short-term outcomes and complications. We also will identify risk factors for experiencing repeated UEs in the NICU, describe the outcomes of those patients with more than one UE, and evaluate longer-term outcomes in these infants. To our knowledge, this is the largest cohort study focusing on risk factors and outcomes associated with UEs, and the only study focusing specifically on repeated UEs.

\section{Methods}

\section{Study population}

We performed a cohort study of all NICU patients who had at least one UE over a 5-year period at Nationwide Children's Hospital in Columbus, Ohio. Inclusion criteria are all infants admitted to the NICU receiving invasive mechanical ventilation via ETT who experienced a UE between January 1, 2014 and December 31, 2018. The only exclusion criteria was if the patient had an unplanned removal of a tracheostomy tube. This study was approved by the Nationwide Children's Hospital Institutional Review Board. The NICU at Nationwide Children's Hospital is a 130-bed, level IV referral center [12]. Our NICU cares for a wide range of patients who might require prolonged mechanical ventilation, including those with extreme prematurity, surgical issues, congenital malformations, and a 24-bed referral center for infants with severe bronchopulmonary dysplasia (BPD). To optimize neurodevelopmental outcomes, routine use of restraints and sedation to prevent UEs is discouraged. The patients were enrolled prospectively and identified by an institutional quality improvement project, in which the Nationwide Children's NICU has been tracking all UEs since January 2014. Retrospective chart review was used to collect additional clinical and demographic data.

\section{Definitions}

We defined a UE as the unintended displacement of the ETT without an order from the provider. Underlying causes identified for UEs include events related to direct provider contact or patient maneuvering, events during holding by family, loose ETT securement device or tape, events related to emesis, and unwitnessed events assumed to be due to patient movement or other unidentified causes. If the ETT was not visibly displaced based on inappropriate depth or loose securement device, a UE was identified by lack of appropriate color change on end-tidal carbon dioxide detector, lack of vital sign improvement with positivepressure ventilation via ETT, and lack of breath sounds on auscultation. Information about each UE and the reintubation procedure were collected from the medical record and from a UE huddle form completed by bedside providers immediately following the event. Completion of the UE huddle form is the primary method of identification of UE events, and capture of all events is further ensured through multidisciplinary discussion at regular meetings and manual chart review. Reporting of UEs was monitored by the quality improvement committee, and multiple interventions were implemented at the start of the project to optimize identification of all UEs.

Short-term outcomes following UE were selected a priori and were pulled from procedure notes, respiratory therapist documentation, and the medication administration record. Facial or airway trauma was defined as documentation of airway edema or erythema noted during direct laryngoscopy, trauma or bleeding to mucous membranes documented in the intubation procedure note, or nursing notes describing mucous membrane injury following the reintubation. For those patients who remained extubated, stridor or hoarse cry documented in the $6 \mathrm{~h}$ following UE was noted. Baseline oxygen requirement is defined as the most frequent fraction of inspired oxygen $\left(\mathrm{FiO}_{2}\right)$ in the $12 \mathrm{~h}$ period before or after the UE. Change in ETT depth is determined by comparing the most recent ETT depth charted before the UE and the depth documented after x-ray confirmation of appropriate depth following reintubation. Appropriateness of the ETT size is determined using the Sherman ratio, which is defined as ETT internal diameter divided by gestational age, with a value less than 0.1 considered acceptable [13]. Flowsheet data were pulled for each patient to determine the total number of different ETTs placed during the NICU stay, the size of each ETT, and the total duration of intubation. Longer-term outcomes at the time of NICU discharge or transfer were defined a priori and were extracted from medical record documentation and problem lists. BPD is defined as the need for supplemental oxygen or respiratory support at 36 weeks postmenstrual age (PMA) in those infants born at less than 32 weeks or at 56 days of life in those born at greater than 32 weeks [14, 15]. A diagnosis of acquired SGS was determined by problem lists and documentation from procedure notes. Acquired SGS severity was classified using the Cotton-Meyer system [16], as documented in the direct laryngoscopy and bronchoscopy 
Table 1 Overall patient demographics and comparison between $1 \mathrm{UE}$ and $\geq 2 \mathrm{UE}$ groups.

\begin{tabular}{|c|c|c|c|c|}
\hline Parameter & Total $(n=300)$ & $1 \mathrm{UE}(n=167)$ & $\geq 2 \operatorname{UE}(n=133)$ & $P$ value* \\
\hline Male sex & $183(61.0 \%)$ & $96(57.5 \%)$ & $87(65.4 \%)$ & 0.19 \\
\hline $\begin{array}{l}\text { Gestational age at } \\
\text { birth (weeks) }\end{array}$ & $26.7(24.6 ; 33.1)$ & $28.0(25.4 ; 37.0)$ & $25.4(24.0 ; 27.9)$ & $<0.0001$ \\
\hline $\begin{array}{l}\text { Postmenstrual age at } \\
\text { first UE (weeks) }\end{array}$ & $34.3(28.8 ; 39.8)$ & $36.4(30.4 ; 40.1)$ & $32.1(27.7 ; 38.6)$ & 0.003 \\
\hline Birth weight (g) & $859.0(615.0 ; 1964.0)$ & $1069.0(695.0 ; 2490.0)$ & $743.0(583.0 ; 982.5)$ & $<0.0001$ \\
\hline $\begin{array}{l}\text { Intrauterine growth } \\
\text { restriction }\end{array}$ & $65(21.7 \%)$ & $33(19.8 \%)$ & $32(24.1 \%)$ & 0.40 \\
\hline Weight at first UE (g) & $1775.0(999.8 ; 2967.5)$ & $2220.0(1187.5 ; 3275.0)$ & $1280.0(885.0 ; 2640.0)$ & 0.0002 \\
\hline Day of life at first UE & $25.4(8.6 ; 65.8)$ & $19.3(6.6 ; 62.5)$ & $32.9(13.5 ; 69.3)$ & 0.01 \\
\hline $\begin{array}{l}\text { Day of life at } \\
\text { admission to } \\
\text { Nationwide } \\
\text { Children's NICU }\end{array}$ & $12.9(34.6)$ & $7.9(25.1)$ & $19.1(43.1)$ & 0.02 \\
\hline Length of stay (days) & $147.0(90.0 ; 214.0)$ & $107.0(48.0 ; 180.0)$ & $191.5(137.8 ; 283.0)$ & $<0.0001$ \\
\hline $\begin{array}{l}\text { ETT days during } \\
\text { NICU stay }\end{array}$ & $41.0(15.0 ; 85.5)$ & $21.0(6.0 ; 56.0)$ & $62.0(34.0 ; 118.0)$ & $<0.0001$ \\
\hline $\begin{array}{l}\text { Number of ETTs } \\
\text { during NICU stay }\end{array}$ & $3(2 ; 6)$ & $2(1 ; 4)$ & $6(4 ; 8)$ & $<0.0001$ \\
\hline
\end{tabular}

Bold values indicate statistical significance $p<0.05$.

Data are expressed as $n(\%)$, median (IQR), or mean (SD).

$U E$ unplanned extubation, $N I C U$ neonatal intensive care unit, ETT endotracheal tube, IQR interquartile range, $S D$ standard deviation.

$* P$ value compares $1 \mathrm{UE}$ vs. $\geq 2 \mathrm{UE}$ groups. procedure note at the time of diagnosis. Data regarding surgical interventions for acquired SGS were extracted from operation notes and procedure codes.

\section{Statistical analysis}

Data were analyzed using GraphPad Prism 8.0.0, IBM SPSS Statistics (Version 26), and R (Version 1.3.5033). Categorical variables were compared using $\chi^{2}$ or Fisher's exact test. Continuous variables were examined using Mann-Whitney $U$ or Kruskal-Wallis test. Multivariable and univariate logistic regression models were used for all outcomes to determine associations between those listed and the odds of having 2 or more UEs and the odds of receiving a diagnosis of acquired SGS. Inverse probability of treatment weighting (IPTW) was utilized as a method to create unbiased comparisons between UE events and diagnosis of acquired SGS. One multivariable logistic regression model was used to assess the odds of having 1 UE vs. 2 or more UEs, while another was created to assess the odds of having a diagnosis of acquired SGS vs. not having a diagnosis of acquired SGS. Variables included in each model to determine propensity scores and gain stabilized weights were determined a priori and included those events that happened prior to UE or receiving a diagnosis of acquired SGS. These variables included sex, gestational age, and birth weight. Standardized mean differences
(SMD) with a threshold of $>10 \%$ were used to determine clinically meaningful differences between covariates and outcomes to include in the model. SMDs were calculated prior to and after weighting. Weighted multivariable logistic regression models were created to determine odds of having 1 UE vs. 2 or more UEs and having a diagnosis of acquired SGS vs. not having a diagnosis of acquired SGS, conditioned on covariates balanced after weighting (SMDs $<10 \%$ threshold) and ETT days. A $p$ value of $<0.05$ was considered statistically significant.

\section{Results}

\section{Study population}

During this study period, our NICU had $\sim 850$ admissions per year. Among these patients, we had an average of 5243 ETT days per year. The UE rate in our NICU at the start of this project was $3.3 \mathrm{UE}$ events per 100 ETT days and decreased to 2.0 UE events per 100 ETT days with quality improvement interventions approximately halfway through the study period. Our baseline rates of BPD during this study period ranged between 46 and 69\%. During our 5year study period, there were 588 UEs among 300 patients. At least 2 UEs were experienced by 133 (44.3\%) of these patients. Of these 300 patients, $183(61.0 \%)$ are male. 
Table 2 Events surrounding each UE event and short-term complications.

\begin{tabular}{|c|c|}
\hline Parameter $(n=588)$ & Value \\
\hline \multicolumn{2}{|l|}{ ETT securement method } \\
\hline Standard commercial device & $569(96.8 \%)$ \\
\hline Tape & $18(3.1 \%)$ \\
\hline Nasal intubation & $1(0.2 \%)$ \\
\hline \multicolumn{2}{|l|}{ Type of ventilation at time of UE } \\
\hline Conventional & $541(92.0 \%)$ \\
\hline High frequency oscillatory ventilation & $47(8.0 \%)$ \\
\hline Skin-to-skin care or holding at time of UE & $96(16.3 \%)$ \\
\hline \multicolumn{2}{|l|}{ Primary cause identified } \\
\hline Direct provider contact & $234(39.8 \%)$ \\
\hline Unwitnessed & $23(3.9 \%)$ \\
\hline Emesis & $98(16.7 \%)$ \\
\hline ETT re-taping & $35(6.0 \%)$ \\
\hline Loose securement device or tape & $125(21.3 \%)$ \\
\hline Unknown & $73(12.4 \%)$ \\
\hline \multicolumn{2}{|l|}{ Time of day } \\
\hline Day shift (7 a.m.-7 p.m.) & $322(54.8 \%)$ \\
\hline Night shift (7 p.m.-7 a.m.) & $266(45.2 \%)$ \\
\hline \multicolumn{2}{|l|}{ Day of week } \\
\hline Monday & $81(13.8 \%)$ \\
\hline Tuesday & $83(14.1 \%)$ \\
\hline Wednesday & $85(14.5 \%)$ \\
\hline Thursday & $87(14.8 \%)$ \\
\hline Friday & $84(14.3 \%)$ \\
\hline Saturday & $84(14.3 \%)$ \\
\hline Sunday & $84(14.3 \%)$ \\
\hline \multicolumn{2}{|l|}{ Feeds held } \\
\hline None/already NPO & $516(87.8 \%)$ \\
\hline $1-3$ feeds & $60(10.2 \%)$ \\
\hline Made NPO & $12(2.0 \%)$ \\
\hline \multicolumn{2}{|l|}{ Resuscitation } \\
\hline None & $328(55.8 \%)$ \\
\hline Bag-mask ventilation to stabilize & $242(41.2 \%)$ \\
\hline Chest compressions & $14(2.4 \%)$ \\
\hline Other $^{\mathrm{a}}$ & $4(0.7 \%)$ \\
\hline Death immediately following UE & $2(0.3 \%)$ \\
\hline \multicolumn{2}{|l|}{ Airway trauma } \\
\hline None & $527(89.6 \%)$ \\
\hline Mucous membrane bleeding & $20(3.4 \%)$ \\
\hline Airway edema/erythema & $33(5.6 \%)$ \\
\hline Stridor and/or hoarse cry & $8(1.4 \%)$ \\
\hline Reintubated within $60 \mathrm{~min}$ of UE & $470(79.9 \%)$ \\
\hline Trial of extubation before reintubation $(n=470)$ & $15(3.2 \%)$ \\
\hline \multicolumn{2}{|l|}{ Medications received for reintubation $(n=470)$} \\
\hline None & $304(64.7 \%)$ \\
\hline Opiate alone & $26(5.5 \%)$ \\
\hline
\end{tabular}

Table 2 (continued)

\begin{tabular}{|c|c|}
\hline Parameter $(n=588)$ & Value \\
\hline Benzodiazepine alone & $101(21.5 \%)$ \\
\hline Opiate and benzodiazepine & $10(2.1 \%)$ \\
\hline Chloral hydrate & $4(0.9 \%)$ \\
\hline Sedation plus neuromuscular blocker & $25(5.3 \%)$ \\
\hline \multicolumn{2}{|l|}{ Number of reintubation attempts $(n=470)$} \\
\hline 1 & $274(58.3 \%)$ \\
\hline 2 & $113(24.0 \%)$ \\
\hline 3 & $50(10.6 \%)$ \\
\hline$\geq 4$ & $33(7.0 \%)$ \\
\hline $\begin{array}{l}\text { Otolaryngology or anesthesia required for reintubation } \\
(n=470)\end{array}$ & $8(1.7 \%)$ \\
\hline \multicolumn{2}{|c|}{ Change to baseline oxygen requirement after reintubation $(n=470)$} \\
\hline Lower & $69(14.7 \%)$ \\
\hline No change & $217(46.2 \%)$ \\
\hline Increased $1-10 \%$ & $136(28.9 \%)$ \\
\hline Increased $>10 \%$ & $48(10.2 \%)$ \\
\hline \multicolumn{2}{|l|}{ Depth of new ETT compared to previous $(n=470)$} \\
\hline Less deep & $32(6.8 \%)$ \\
\hline Same depth & $230(48.9 \%)$ \\
\hline $0.5-1 \mathrm{~cm}$ deeper & $173(36.8 \%)$ \\
\hline$>1 \mathrm{~cm}$ deeper & $10(2.1 \%)$ \\
\hline Previous depth unknown or not documented & $25(5.3 \%)$ \\
\hline \multicolumn{2}{|l|}{ Reintubation alternative $(n=118)$} \\
\hline Room air & $13(11.0 \%)$ \\
\hline Low flow nasal cannula & $6(5.1 \%)$ \\
\hline High flow nasal cannula & $10(8.5 \%)$ \\
\hline Continuous positive airway pressure & $89(75.4 \%)$ \\
\hline
\end{tabular}

Data are expressed as $n(\%)$.

$U E$ unplanned extubation, ETT endotracheal tube, NPO nil per os.

${ }^{a}$ Including treatment for bronchospasm and pulmonary hemorrhage.

Demographic information for these patients is described in Table 1 . The majority of patients in this overall cohort were premature, with 217 (72.3\%) born at less than 32 weeks gestation. These patients tended to have a long duration of intubation and long length of hospital stay, with 174 (58.0\%) having greater than 30 ETT days and 173 (57.7\%) having a length of stay in the NICU greater than 120 days. As shown in Table 1, the patients who experienced a single $\mathrm{UE}$ and those who experienced repeated events were quite different at baseline.

\section{Events surrounding UE}

Details about the events surrounding each of the 588 UE events and potential causes or contributing factors are listed in Table 2. Of note, $16.3 \%$ percent of the UEs occurred during skin-to-skin care or holding by family members and $39.8 \%$ 
Table 3 Number of patients experiencing adverse short-term outcomes following any UE.

\begin{tabular}{lccc}
\hline Parameter & 1 UE $(n=167)$ & $\geq 2$ UE $(n=133)$ & $P$ value \\
\hline Being held at time of any UE & $27(16.2 \%)$ & $50(37.6 \%)$ & $<\mathbf{0 . 0 0 0 1}$ \\
Any number of feeds held after a UE & $22(13.2 \%)$ & $38(28.6 \%)$ & $\mathbf{0 . 0 0 1}$ \\
Made NPO after a UE & $7(4.2 \%)$ & $4(3.0 \%)$ & 0.76 \\
Required any resuscitation & $45(26.9 \%)$ & $104(78.2 \%)$ & $<\mathbf{0 . 0 0 0 1}$ \\
Required bag-mask ventilation & $45(26.9 \%)$ & $104(78.2 \%)$ & $<\mathbf{0 . 0 0 0 1}$ \\
Required chest compressions & $3(1.8 \%)$ & $11(8.3 \%)$ & $\mathbf{0 . 0 1}$ \\
Any documented airway trauma & $18(10.8 \%)$ & $35(26.3 \%)$ & $\mathbf{0 . 0 0 1}$ \\
Any medication administered for reintubation & $28(16.8 \%)$ & $60(45.1 \%)$ & $<\mathbf{0 . 0 0 0 1}$ \\
Any benzodiazepine given for reintubation & $8(4.8 \%)$ & $48(36.1 \%)$ & $<\mathbf{0 . 0 0 0 1}$ \\
Any opiate given for reintubation & $28(16.8 \%)$ & $25(18.8 \%)$ & 0.65 \\
Any neuromuscular blocker given for reintubation & $12(7.2 \%)$ & $14(10.5 \%)$ & 0.31 \\
Ever reintubated within 60 min of UE & $100(59.9 \%)$ & $91(68.4 \%)$ & 0.15 \\
Ever needed $>1$ attempt at reintubation & $35(21.0 \%)$ & $99(74.4 \%)$ & $<\mathbf{0 . 0 0 0 1}$ \\
Ever needed $>2$ attempts at reintubation & $15(9.0 \%)$ & $55(41.4 \%)$ & $<\mathbf{0 . 0 0 0 1}$ \\
Ever needed otolaryngology or anesthesia for reintubation & $1(0.6 \%)$ & $5(3.8 \%)$ & 0.09 \\
Ever had baseline oxygen requirement increased by $>10 \%$ & $33(19.8 \%)$ & $44(33.1 \%)$ & $\mathbf{0 . 0 1}$ \\
after UE & & & \\
\hline Bold values indicate statistical significance $p<0.05$. & & & \\
Data are expressed as $N$ (\%). & & & \\
$U E$ unplanned extubation, $N P O$ nil per os. & &
\end{tabular}

were attributed to direct provider contact or maneuvering. Short-term complications and outcomes in the period immediately following the UE are detailed in Table 2. Resuscitation, including bag-mask ventilation, cardiopulmonary resuscitation with chest compressions, and treatment for pulmonary hemorrhage or bronchospasm, was needed following $260(44.2 \%)$ of the UEs. Additionally, two (0.3\%) of the UEs resulted in death. Of the 470 events that led to reintubation within $60 \mathrm{~min}$ of UE, $166(35.3 \%)$ received sedation or paralytic medications and $196(41.7 \%)$ needed more than one attempt at reintubation. In 183 (38.9\%) events, the appropriate position of the new ETT following reintubation was noted to be deeper than the previous tube had been.

Table 3 compares the number of patients who experienced specific adverse events after at least one UE. A significantly greater number of patients in the $\geq 2$ UE group were exposed to short-term adverse events related to a UE. More patients in the $\geq 2$ UE group had feeds held after a UE, required resuscitation including bag-mask ventilation or chest compressions, had airway trauma associated with a UE, received sedative medications for reintubation, required multiple attempts at intubation, and had an increase in baseline oxygen requirement of $>10 \%$ following a UE.

\section{Repeated UE}

Repeated UEs were experienced by 133 (44.3\%) of patients included in this study. The group that experienced multiple UEs had a lower median gestational age at birth and PMA at the time of UE, but experienced the first UE at a significantly older day of life (DOL). The multiple UE group also had a significantly higher total number of ETT days during the initial NICU admission. Table 4 compares patients who experienced a single UE with those who experienced at least two. Crude, adjusted, and weighted and adjusted odds ratios are shown to compare clinical characteristics between the two groups and identify factors that are associated with a higher odds of experiencing repeat UEs. Longer-term outcomes were also compared between the two groups. Patients who received a diagnosis of BPD had significantly increased odds (weighted and adjusted OR 6.78, $p=0.02$ ) of experiencing more than one UE compared to those with no BPD diagnosis. Significantly increased odds of having a length of stay greater than 90 days (weighted and adjusted OR 3.12, $p=0.04$ ) and having a tracheostomy placed during admission (weighted and adjusted OR 4.16, $p=0.002$ ) were also seen in those with more than one UE.

Table 2 shows the respiratory outcomes following UE. Of the 470 events leading to reintubation within $60 \mathrm{~min}$, only $15(3.2 \%)$ were given a trial of extubation. Of the events leading to reintubation within $60 \mathrm{~min}, 184(39.1 \%)$ led to an increase in oxygen requirement between 1 and $49 \%$ above baseline, with a median increase of $5 \%$ (interquartile range (IQR) 3-11\%). Following the 118 events that did not lead to reintubation within $60 \mathrm{~min}$, the majority required continuous positive airway pressure (CPAP), but $16(13.6 \%)$ only needed nasal cannula and $13(11.0 \%)$ remained stable in room air. 
Table 4 Characteristics and outcomes of those patients with $\geq 2$ UEs compared to those with 1 UE.

\begin{tabular}{|c|c|c|c|c|c|c|}
\hline Parameter & $\begin{array}{l}1 \mathrm{UE} \\
(n=167)\end{array}$ & $\begin{array}{l}\geq 2 \mathrm{UE} \\
(n=133)\end{array}$ & Crude OR & Adjusted $\mathrm{OR}^{\mathrm{a}}$ & $\begin{array}{l}\text { Weighted and adjusted } \\
\mathrm{OR}^{\mathrm{b}}\end{array}$ & $P$ value* \\
\hline Weight at first $\mathrm{UE}<2000 \mathrm{~g}$ & $75(44.9 \%)$ & $86(64.7 \%)$ & $2.24(1.41 ; 3.60)$ & $1.28(0.68,2.38)$ & $1.29(0.69,2.40)$ & 0.42 \\
\hline PMA at first $\mathrm{UE}<30$ weeks & $39(23.4 \%)$ & $55(41.4 \%)$ & $2.31(1.41 ; 3.83)$ & $1.74(0.92,3.31)$ & $2.01(1.06,3.83)$ & $\mathbf{0 . 0 3}$ \\
\hline DOL at first $\mathrm{UE}<30$ days & $89(53.3 \%)$ & $72(54.1 \%)$ & $1.03(0.66 ; 1.64)$ & $0.88(0.52,1.48)$ & $0.83(0.47,1.44)$ & 0.50 \\
\hline Length of stay $>90$ days & $101(60.5 \%)$ & $120(90.2 \%)$ & $7.64(3.87 ; 16.45)$ & $2.21(0.92,5.69)$ & $3.12(1.08,9.03)$ & 0.04 \\
\hline Critical airway & $6(3.6 \%)$ & $3(2.3 \%)$ & $0.62(0.13 ; 2.39)$ & $2.09(0.35,11.17)$ & $1.31(0.26,6.68)$ & 0.75 \\
\hline $\begin{array}{l}\text { Chromosomal anomaly/genetic } \\
\text { syndrome }^{c}\end{array}$ & $18(10.8 \%)$ & $15(11.3 \%)$ & $1.05(0.51 ; 2.17)$ & $1.89(0.66,5.59)$ & $2.32(0.72,7.47)$ & 0.16 \\
\hline $\begin{array}{l}\text { Mortality during initial } \\
\text { hospitalization }\end{array}$ & $22(13.2 \%)$ & $14(10.5 \%)$ & $0.81(0.39 ; 1.63)$ & $0.41(0.16,0.99)$ & $0.41(0.16,1.08)$ & 0.07 \\
\hline Bronchopulmonary dysplasia & $96(57.5 \%)$ & $119(89.5 \%)$ & $7.81(3.96 ; 16.92)$ & $3.08(0.88,11.73)$ & $6.78(1.42,32.31)$ & $\mathbf{0 . 0 2}$ \\
\hline Tracheostomy & $18(10.8 \%)$ & $35(26.3 \%)$ & $2.96(1.59 ; 5.61)$ & $3.03(1.38,6.95)$ & $4.16(1.68,10.31)$ & 0.002 \\
\hline
\end{tabular}

Bold values indicate statistical significance $p<0.05$.

Data are expressed as $N(\%)$ or odds ratio (95\% confidence interval).

$U E$ unplanned extubation, $O R$ odds ratio, $P M A$ postmenstrual age, $D O L$ day of life, $I P T W$ inverse probability of treatment weighting.

* $P$ values are displayed from weighted and adjusted models.

${ }^{a}$ Adjusted odds ratios displayed as adjusted for sex, gestational age, birth weight, and total number of ETT days during initial hospitalization.

${ }^{b}$ Weighted and adjusted ORs are IPTW weighted for sex, gestational age, and birth weight; and adjusted for sex, gestational age, birth weight, and total number of ETT days during initial hospitalization.

'Including Trisomy 21, Stickler syndrome, CHARGE syndrome, Wolff-Hirschhorn syndrome, DiGeorge syndrome, and a chromosome 19 anomaly.

\section{Outcomes at NICU discharge}

On assessment of outcomes at the time of NICU discharge or transfer to another facility for these 300 patients, 36 (12.0\%) died during their initial NICU stay. Two deaths were directly following the UE; in both cases, the babies had a modified Allow Natural Death order and were not reintubated due to parental preference for comfort care. Of these 300 infants, 215 (71.7\%) were diagnosed with BPD and $53(17.7 \%)$ required tracheostomy placement. In addition to those requiring home ventilatory support via tracheostomy, 129 (43.0\%) were discharged with nasal cannula oxygen and $3(1.0 \%)$ required CPAP at home.

\section{Acquired subglottic stenosis}

Of these 300 patients, $28(9.3 \%)$ were diagnosed with acquired SGS. The severity of acquired SGS was classified as Cotton-Myer grade 1 in 9 patients, grade 2 in 6, the most severe grade 3 in 9, and not graded in the remaining 4 patients. The crude, adjusted, and weighted and adjusted odds ratios comparing those who were diagnosed with acquired SGS and those who were not are detailed in Table 5. Significantly fewer patients in the group diagnosed with acquired SGS were born at less than 25 weeks' gestational age $(3.5 \%$ vs $11.3 \%, p=0.043)$. After weighting and adjusting for sex, gestational age at birth, birth weight, and total number of ETT days, characteristics associated with a significantly increased odds of having acquired SGS were having at least four UEs, having documented airway trauma during an intubation attempt, having a history of tracheitis, and having a diagnosis of a major chromosomal anomaly or syndrome. Experiencing the first UE at a weight of $<1500 \mathrm{~g}$ was associated with a decreased odds of having a diagnosis of acquired SGS. When the subgroup with severe acquired SGS requiring surgical intervention, including balloon dilations and laryngotracheal reconstruction, was compared to the group with no SGS or mild SGS not requiring surgery, no significant differences were seen between the two groups.

The median age of acquired SGS diagnosis in this cohort was 3.6 months (IQR 2.8-8.5), and all but one case was diagnosed by 14 months of life. The initial presentation leading to diagnosis of SGS was stridor in 10 of the patients and inability to extubate after multiple attempts in 12 of the patients, with the remainder diagnosed incidentally on direct laryngoscopy and bronchoscopy for another clinical indication. Surgical intervention was required in 13 (46.4\%) of the patients with acquired SGS, including balloon dilations in $8(28.6 \%)$ patients and laryngotracheal reconstruction in $5(17.9 \%)$ patients. The median age at initial SGS surgery was 4 months (IQR 3-10).

\section{Discussion}

UE requiring reintubation is among the most common adverse safety events reported in North American NICUs [2]. 
Table 5 Characteristics of those patients with acquired SGS compared to those without acquired SGS.

\begin{tabular}{|c|c|c|c|c|c|c|}
\hline Outcomes & $\begin{array}{l}\text { No SGS } \\
(n=272)\end{array}$ & $\begin{array}{l}\text { SGS } \\
(n=28)\end{array}$ & Crude OR & Adjusted $\mathrm{OR}^{\mathrm{a}}$ & $\begin{array}{l}\text { Weighted and adjusted } \\
\mathrm{OR}^{\mathrm{b}}\end{array}$ & $P$ value* \\
\hline $\begin{array}{l}\text { Postmenstrual age at first UE }< \\
32 \text { weeks }\end{array}$ & $116(42.6 \%)$ & $6(21.4 \%)$ & $0.37(0.13,0.88)$ & $0.44(0.15,1.19)$ & $0.40(0.15,1.07)$ & 0.07 \\
\hline Weight at first $\mathrm{UE}<1500 \mathrm{~g}$ & $128(47.1 \%)$ & $7(25.0 \%)$ & $0.38(0.14,0.87)$ & $0.37(0.13,0.96)$ & $0.34(0.13,0.91)$ & $\mathbf{0 . 0 3}$ \\
\hline DOL at first $\mathrm{UE}<21$ days & $122(44.9 \%)$ & $11(39.3 \%)$ & $0.80(0.35,1.74)$ & $0.77(0.34,1.7)$ & $0.73(0.32,1.67)$ & 0.46 \\
\hline$\geq 4$ UEs & $30(11.0 \%)$ & $8(28.6 \%)$ & $3.23(1.25,7.75)$ & $3.54(1.19,10.07)$ & $4.25(1.35,13.45)$ & 0.01 \\
\hline $\begin{array}{l}\geq 3 \text { reintubation attempts } \\
\text { following any UE }\end{array}$ & $63(23.2 \%)$ & $7(25.0 \%)$ & $1.11(0.42,2.61)$ & $0.97(0.36,2.36)$ & $0.91(0.36,2.27)$ & 0.83 \\
\hline$\geq 4$ ETTs during hospitalization & $124(45.6 \%)$ & $19(67.9 \%)$ & $2.52(1.13,6.03)$ & $2.22(0.77,6.68)$ & $2.79(0.98,7.93)$ & 0.06 \\
\hline Sherman ratio $\geq 0.1^{\mathrm{c}}$ for any ETT & $20(7.4 \%)$ & $1(3.6 \%)$ & $0.86(0.05,4.92)$ & $0.95(0.05,5.77)$ & $0.94(0.15,5.98)$ & 0.95 \\
\hline Airway trauma with reintubation & $41(15.1 \%)$ & $12(42.9 \%)$ & $4.23(1.83,9.56)$ & $4.31(1.81,10.19)$ & $5.66(2.15,14.87)$ & $<0.001$ \\
\hline Tracheitis & $35(12.9 \%)$ & $18(64.3 \%)$ & $12.19(5.31,29.53)$ & $12.4(4.99,32.81)$ & $13.16(5.04,34.35)$ & $<0.001$ \\
\hline $\begin{array}{l}\text { Chromosomal anomaly/genetic } \\
\text { syndrome }^{\mathrm{d}}\end{array}$ & $25(9.2 \%)$ & $8(28.6 \%)$ & $3.95(1.51,9.65)$ & $4.55(1.44,14.26)$ & $5.62(1.49,21.13)$ & 0.01 \\
\hline
\end{tabular}

Bold values indicate statistical significance $p<0.05$.

Data are expressed as $N(\%)$ or odds ratio (95\% confidence interval).

$S G S$ subglottic stenosis, $O R$ odds ratio, $U E$ unplanned extubation, $D O L$ day of life, ETT endotracheal tube, IPTW inverse probability of treatment weighting.

* $P$ values are displayed from weighted and adjusted models.

${ }^{a}$ Adjusted odds ratios displayed as adjusted for sex, gestational age, birth weight, and total number of ETT days during initial hospitalization.

${ }^{\mathrm{b}}$ Weighted and adjusted ORs are IPTW weighted for sex, gestational age, and birth weight; and adjusted for sex, gestational age, birth weight, and total number of ETT days during initial hospitalization.

${ }^{\mathrm{c}}$ Sherman ratio $=$ ETT size/gestational age.

${ }^{\mathrm{d}}$ Trisomy 21, Stickler syndrome, CHARGE syndrome, Wolff-Hirschhorn syndrome, DiGeorge syndrome, and a chromosome 19 anomaly.

In this study spanning 5 years, we describe factors surrounding UEs, short-term outcomes, and longer-term morbidities in a level IV NICU. UEs in general were associated with many short-term complications such as increase in baseline oxygen requirement, missed feeds, airway trauma, and need for cardiopulmonary resuscitation. Repeated UEs were common among this population and were associated with increased odds of longer-term morbidities, including BPD, need for tracheostomy, and a longer length of stay.

Previous studies support the idea that standardizing the ETT securement leads to fewer UEs [1, 17]. In agreement with these studies, the standard practice in our unit is to secure the ETT using a commercial ETT holder, and this device was used in the vast majority of these events. Skinto-skin care is strongly encouraged in our NICU, and we found that $16 \%$ of the events occurred during skin-to-skin care or holding, which higher than rates reported in other studies $[18,19]$. This finding prompted further quality improvement interventions to ensure airway safety.

Previous studies have shown mixed results regarding the association of weight and PMA with UE. One study showed that most events happened at $<31$ weeks PMA, but others showed that most patients were $>34$ weeks $[8,18,20]$. In our study, the patients had a median birth gestational age of 26.7 weeks, but the UEs happened at a median PMA of
34.3 weeks. Our patients had a median weight of $1775 \mathrm{~g}$ at the time of UE, whereas other studies have shown the majority of UEs occurring in smaller babies [3, 21]. Older and larger patients may be more difficult to contain and warrant creative non-pharmacologic interventions to maintain airway safety.

Several short-term complications were noted as a direct result of the UEs, including increased oxygen requirement and discontinued feeds. About 10\% had documented airway trauma and almost half of patients who were reintubated required multiple attempts. Notably 14 events led to chest compressions and $41.2 \%$ required bag-mask ventilation to stabilize vital signs before a reintubation attempt was made. These short-term complications likely contribute to an increased length of stay and long-term morbidities in these patients, reinforcing the need to focus efforts on UE prevention. Many patients received sedative or paralytic medications for reintubation. In our efforts to improve the potential neurodevelopmental outcome of the patients, our unit discourages the routine use of sedation or restraints to prevent UE. The use of sedation in the setting of reintubation may also negatively impact neurodevelopment. When specifically comparing the single UE group to those patients who experienced multiple UEs, there was greater exposure to many adverse events among the patients with 
multiple UEs, putting these patients at greater risk for shortand longer-term morbidities.

We documented reintubation within $60 \mathrm{~min}$ based on the definitions for UE tracking provided by the Solutions for Patient Safety collaborative. We noted reintubation within $60 \mathrm{~min}$ of UE in $\sim 80 \%$ of the events. Previous studies have reported reintubation rates within $24 \mathrm{~h}$ after UE ranging from 25 to $75 \%$ [18, 20-22]. Our higher rate suggests that many of our intubated patients do require invasive mechanical ventilation, but there is still an opportunity to improve identification of extubation readiness, given that $20 \%$ did not need to be reintubated. In addition, as shown in Table 2, 25\% of these patients did not even require CPAP following UE. Thirty-nine percent of ETTs were replaced deeper than they had previously been taped, indicating a need to continually reassess tube depth, especially in patients who may experience significant growth while intubated due to edema or prolonged duration of intubation.

Although previous studies have reported on risk factors for UEs and subsequent outcomes in the NICU, to our knowledge, none have specifically assessed those patients who experience repeated events. In our study, almost half of the patients had more than one UE. Patients with multiple UEs had a longer duration of intubation on average, so they are already at increased risk for airway and pulmonary morbidities due to prolonged mechanical ventilation. Repeated UEs were seen more often in those patients with BPD, likely due to the prolonged duration of intubation increasing the number of opportunities for UE. In those patients with repeated UEs, higher odds of tracheostomy requirement and home oxygen or ventilator requirement were noted. Care should be taken to prevent UEs in these patients to avoid further increasing the risk for airway trauma and complications.

Previous studies have identified an association between UEs and the development of SGS [11]. In our study population, all but one case of acquired SGS was diagnosed before 14 months of life, with the majority in the first few months of life. The most common clinical presentations leading to this diagnosis were stridor and inability to extubate, so further evaluation for SGS should be considered in this population of patients. Acquired SGS should also be considered in patients following discharge who present with stridor and a history of intubation and UE. Other identified risk factors for acquired SGS in this study include documented airway trauma with reintubation, history of tracheitis, and at least four UEs. We saw a trend toward a greater number of ETTs overall being associated with the development of acquired SGS, which is consistent with previous studies [23-25].

Gestational age has been inconsistently associated with acquired SGS, with some older studies showing no association and a more recent study demonstrating high rates in infants born at 23-24 weeks [11, 23, 26]. Our data actually demonstrated a significantly decreased incidence of acquired SGS in this population of infants born at less than 25 weeks' gestation, potentially related to permissive air leak in our unit and general avoidance of elective ETT upsizing. The Sherman ratio (ETT internal diameter divided by gestational age) has been used in previous studies to assess the appropriateness of ETT size with a ratio of $<0.1$ considered acceptable [23]. Unlike in previous studies $[23,27]$, we found no significant association between Sherman ratio and development of acquired SGS. A recent study reported an overall incidence of $0.93 \%$ of severe acquired SGS in intubated neonates, defined as those requiring surgical intervention to manage SGS;[11] our rate in this study of patients with a history of UE is much higher at $4.3 \%$. This higher incidence might be attributable to the airway trauma and multiple intubation attempts seen with reintubation following UE in this population, many of which likely occurred in an urgent or emergent setting.

One limitation of this study is the retrospective nature of some data collection, although the patients were identified prospectively and the forms detailing the situation surrounding the UE are filled out in real-time after the event, which partially mitigates this concern. Similarly, many outcomes of interest listed in this study are taken from documentation in the medical record, which may lead to limited or missing data. This study also represents the experience of a single level IV NICU with an entirely out born population, though patients were admitted relatively early, at 13 days of life on average (median DOL 0; IQR $0-4)$. It is possible that patients experienced a UE at the birth hospital prior to admission to our NICU. Finally, follow up information is not available for all patients following discharge from the NICU, so there may be a greater number of patients diagnosed with long-term morbidities than identified here.

\section{Conclusions}

UEs are a relatively common safety event in the NICU and can lead to serious short- and long-term morbidities, especially when a patient has multiple events. Identification of common clinical scenarios surrounding UEs has helped to identify key areas to target with quality improvement initiatives. Certain patient characteristics were associated with increased rates of repeated UEs and this knowledge may be used to heighten awareness of high-risk patients. Identification of complications associated with UEs can help to reinforce the importance of preventing these events. These patients are at risk for long-term airway morbidities, which may present after the time of NICU discharge. Further initiatives are needed to use these data to decrease overall UE rates and reduce the need for emergent reintubations. 
Additional research is needed to develop a prediction model to identify those patients at highest risk to experience one or multiple UEs to further improve outcomes.

Author contributions LRP, JD, EGS, and MM conceived and designed this research; LRP, JD, and GR collected and analyzed data; EH analyzed data; LRP drafted the manuscript. All authors edited and revised the manuscript. All authors approved the final version of the manuscript.

\section{Compliance with ethical standards}

Conflict of interest The authors declare that they have no conflict of interest.

Publisher's note Springer Nature remains neutral with regard to jurisdictional claims in published maps and institutional affiliations.

\section{References}

1. Merkel L, Beers K, Lewis MM, Stauffer J, Mujsce DJ, Kresch MJ. Reducing unplanned extubations in the NICU. Pediatrics. 2014;133:e1367-72.

2. Sharek PJ, Horbar JD, Mason W, Bisarya H, Thurm CW, Suresh $\mathrm{G}$, et al. Adverse events in the neonatal intensive care unit: development, testing, and findings of an NICU-focused trigger tool to identify harm in North American NICUs. Pediatrics. 2006;118:1332-40.

3. Carvalho FL, Mezzacappa MA, Calil R, Machado, Hda C. Incidence and risk factors of accidental extubation in a neonatal intensive care unit. J Pediatr. 2010;86:189-95.

4. Ligi I, Millet V, Sartor C, Jouve E, Tardieu S, Sambuc R, et al. Iatrogenic events in neonates: beneficial effects of prevention strategies and continuous monitoring. Pediatrics. 2010;126: e1461-1468.

5. Hatch LD, Grubb PH, Lea AS, Walsh WF, Markham MH, Whitney GM, et al. Endotracheal intubation in neonates: a prospective study of adverse safety events in 162 infants. J Pediatr. 2016;168:62-66.e6.

6. Klugman D, Berger JT, Spaeder MC, Wright A, Pastor W, Stockwell DC. Acute harm: unplanned extubations and cardiopulmonary resuscitation in children and neonates. Intensive Care Med. 2013;39:1333-4.

7. Conner GH, Maisels MJ. Orotracheal intubation in the newborn. Laryngoscope. 1977;87:87-91.

8. da Silva PS, Reis ME, Aguiar VE, Fonseca MC. Unplanned extubation in the neonatal ICU: a systematic review, critical appraisal, and evidence-based recommendations. Respir Care. 2013;58:1237-45.

9. Loganathan PK, Nair V, Vine M, Kostecky L, Kowal D, Soraisham A. Quality improvement study on new endotracheal tube securing device (Neobar) in neonates. Indian $\mathbf{J}$ Pediatr. 2017;84:20-24.
10. Kambestad KK, Huack A, Nair S, Chapman R, Chin S, Langga L, et al. The adverse impact of unplanned extubation in a cohort of critically ill neonates. Respir Care. 2019;64:1500-7.

11. Thomas RE, Rao SC, Minutillo C, Vijayasekaran S, Nathan EA. Severe acquired subglottic stenosis in neonatal intensive care graduates: a case-control study. Arch Dis Child Fetal Neonatal Ed. 2018;103:F349-F354.

12. American Academy of Pediatrics Committee on Fetus and Newborn. Levels of neonatal care. Pediatrics. 2012;130:587-97.

13. Sherman JM, Nelson H. Decreased incidence of subglottic stenosis using an "appropriate-sized" endotracheal tube in neonates. Pediatr Pulmonol. 1989;6:183-5.

14. Poindexter BB, Feng R, Schmidt B, Aschner JL, Ballard RA, Hamvas A, et al. Comparisons and limitations of current definitions of bronchopulmonary dysplasia for the prematurity and respiratory outcomes program. Ann Am Thorac Soc. 2015;12:1822-30.

15. Ehrenkranz RA, Walsh MC, Vohr BR, Jobe AH, Wright LL, Fanaroff AA, et al. Validation of the National Institutes of Health consensus definition of bronchopulmonary dysplasia. Pediatrics. 2005; 116:1353-60.

16. Myer CM 3rd, O'Connor DM, Cotton RT. Proposed grading system for subglottic stenosis based on endotracheal tube sizes. Ann Otol Rhinol Laryngol. 1994;103:319-23.

17. Crezee KL, DiGeronimo RJ, Rigby MJ, Carter RC, Patel S. Reducing unplanned extubations in the NICU following implementation of a standardized approach. Respir Care. 2017;62:1030-5.

18. Veldman A, Trautschold T, Weiss K, Fischer D, Bauer K Characteristics and outcome of unplanned extubation in ventilated preterm and term newborns on a neonatal intensive care unit. Paediatr Anaesth. 2006;16:968-73.

19. DeJonge MH, White M. A comparison of two methods of oral endotracheal tube stabilization in neonatal patients. J Perinatol. 1998;18:463-5.

20. Galiote JP, Ridore M, Carman J, Zell L, Brant K, Gayle C, et al. Reduction in unintended extubations in a level IV neonatal intensive care unit. Pediatrics. 2019;143:e20180897.

21. Fontanez-Nieves TD, Frost M, Anday E, Davis D, Cooperberg D, Carey AJ. Prevention of unplanned extubations in neonates through process standardization. J Perinatol. 2016;36:469-73.

22. Loughead JL, Brennan RA, DeJuilio P, Camposeo V, Wengert J, Cooke D. Reducing accidental extubation in neonates. Jt Comm J Qual Patient Saf. 2008;34:164-70.

23. Sherman JM, Lowitt S, Stephenson C, Ironson G. Factors influencing acquired subgottic stenosis in infants. $\mathrm{J}$ Pediatr. 1986;109:322-7.

24. Nicklaus PJ, Crysdale WS, Conley S, White AK, Sendi K, Forte V. Evaluation of neonatal subglottic stenosis: a 3-year prospective study. Laryngoscope. 1990;100:1185-90.

25. Downing GJ, Kilbride HW. Evaluation of airway complications in high-risk preterm infants: application of flexible fiberoptic airway endoscopy. Pediatrics. 1995;95:567-72.

26. Fan LL, Flynn JW, Pathak DR. Risk factors predicting laryngeal injury in intubated neonates. Crit Care Med. 1983;11:431-3.

27. Contencin P, Narcy P. Size of endotracheal tube and neonatal acquired subglottic stenosis. Study group for neonatology and pediatric emergencies in the Parisian area. Arch Otolaryngol Head Neck Surg. 1993;119:815-9. 